\title{
Les marqueurs d'identité masculine et féminine dans les fabliaux
}

\author{
Urh Ferlež, Miha Pintarič
}

\section{Résumé}

L'article traite les marqueurs d'identité dans les fabliaux, vérifiant l'hypothèse selon laquelle les hommes dans les fabliaux sont identifiés par ce qu'ils font, par le métier qu'ils exercent plutôt que par leurs caractéristiques physiques, et que les femmes sont soit identifiées par leur mari soit par leurs caractéristiques à elles. L'analyse qualitative et, ensuite, quantitative des fabliaux montre que la quantité d'informations que le lecteur reçoit sur l'identité d'un personnage varie d'un fabliau à l'autre, que les auteurs ne différencient les genres selon la quantité des marqueurs d'identité, selon leurs caractéristiques physiques, que les personnages masculins principaux sont effectivement souvent caractérisés par leur métier, alors que les personnages féminins, dans la grande majorité des cas, ne portent pas le marqueur du métier, à l'exception des personnages secondaires ou ceux qui ont le même métier.

Mots clés : fabliaux, marqueurs d'identité, hommes/femmes du Moyen âge, Rutebeuf, Jean Bodel 


\section{INTRODUCTION}

Les fabliaux, petits contes transmettant les événements de la vie quotidienne, offrent, dans la clarté de leur univers, une vaste richesse de situations sociales. Dans les fabliaux, l'homme médiéval rêve les mondes imaginaires, tout en s'intéressant à ce qui l'entoure. Et qu'est-ce qui l'entoure ? Ce sont les autres, définis par rapport à l'homme médiéval, qui n'est qu'un « autre » entre les « autres ». À l'époque, le mariage (le couple) était essentiel à l'ordre social. Les récits des fabliaux y attachent un intérêt particulier. Certains personnages possèdent un nom, d'autres en sont privés. Il est clair qu'ils ont un sexe, et cela va de soi qu'ils ont aussi les devoirs qui appartiennent à ce sexe. Une femme lambda et un homme lambda, c'est le début d'un fabliau, mais la question d'identité se pose quand les hommes sont définis par leurs métiers, perdant en quelque sorte leurs caractéristiques masculines tandis que les femmes restent femmes, tout en adoptant, alors, une identité caractérisée par la beauté ou par la ruse. Est-ce toutefois la règle ? Nous essaierons de répondre à cette question.

Depuis la fin du XIX ${ }^{\mathrm{e}}$ siècle, quand ils sont devenus le sujet d'études universitaires, un nombre infini de thèmes des fabliaux ont été traités. Les chercheurs ont analysé les récits sous des angles différents : selon le contenu, en laissant le côté linguistique à part etc. Par exemple, ils y ont cherché le comique (Crocker ; Pearcy), le rôle didactique (Stearns Schenck; Horton) ou les sentiments (Wehner Eaton). Ils se sont concentrés en particulier sur le rôle et la position des femmes, étant donné que les fabliaux illustrent la société médiévale et que celle-ci semble imprégnée de misogynie et de phallocentrisme. Par conséquent, les travaux s'offrant à notre curiosité portent sur la soumission des femmes, prostitution, violence dans l'érotisme, mariage etc. (Muñoz; Bégin ; Collart ; de Looze ; Jacobs).

Le personnage de fabliau en représente un échantillon, appelé «type ». Chaque type est, dans l'histoire, le porteur des valeurs de groupe, de société (Dufournet, 14). Il expose ces valeurs aussi bien que son statut social. Il est facile de distinguer un paysan d'un ecclésiastique et un bourgeois d'un aristocrate. Parfois, cela est clairement marqué, pourtant, dans certains fabliaux, il faut passer par d'autres aspects pour déduire la position du personnage. Dans ces cas-là, le lecteur doit lire attentivement et constater où vit le protagoniste, s'il a des biens en sa possession, comment il se comporte ou même comment il est vêtu (Leech, 83; Stearns Schenck, 71). Jean Dufournet dit que le personnage, chaque type dans les fabliaux, est un héraut qui proclame des valeurs par l'imposition d'un nom, par son habileté, sa ruse, des attributs ou des objets symboliques (Dufournet, 13-16). Il ne s'agit pas de se pencher sur les statuts sociaux dans les fables, ni sur les valeurs dont les personnages sont porteurs. Nous nous poserons la question de l'identité des sexes sans nous attarder sur la misogynie. Nous nous concentrerons sur les signes 
grâce auxquels les personnages sont marqués, identifiés et identifiables au lecteur, et nous essaierons de montrer la différence entre l'identification des personnages masculins et féminins.

Notre hypothèse s'exprime ainsi : les hommes sont identifiés par ce qu'ils font, par le métier qu'ils exercent (ils sont paysans, maçons, prêtres, marchands etc.) et moins souvent par leurs caractéristiques physiques et mentales (beau, gentil, rusé, etc.). Les femmes, elles, sont soit identifiées par leur mari et même cela avec un empêchement ("belle marchande ", par exemple, indique à la fois que la femme est belle et que son mari est marchand), soit par leurs caractéristiques (la beauté, la ruse, l'intelligence, etc.). Nous le vérifierons à travers dix-huit fabliaux choisis. ${ }^{1}$ Nous traiterons les fabliaux dans lesquels les marqueurs d'identité sont exprimés de manière plus complexe ou détaillée, d'autres serviront pour l'analyse quantitative.

\section{BODEL}

Prenons, d'abord, les quatre fabliaux de maître Jean Bodel. Dans le premier, intitulé $D u$ villain de Bailleul, nous trouvons un couple marié et un clerc, ainsi que dans De Gombert et des deus clers et De Brunain et la vache au prestre. Les clercs, évidemment des hommes, y sont aussi nommés chapelain ou sacristain. Ils ne sont pas caractérisés par des épithètes, mais nous pouvons déduire de leurs actions qu'ils sont voluptueux, gloutons, malins et peu fidèles au célibat. Bodel commence son fabliau du paysan de Bailleul avec une description assez longue de ses caractéristiques corporelles et de son tempérament. Il nous fait savoir ce qu'il est (un paysan) et ce qu'il n'est pas (ni usurier, ni banquier). L'écrivain se donne la peine de peindre sa laideur, qui correspond à son caractère effrayant. Le paysan de Bailleul est ainsi caractérisé par un métier et des adjectifs caractéristiques. Sa femme, elle, possède un prénom (Erne) et le lecteur obtient des informations sur son caractère : elle est une dame rouée et astucieuse qui préférerait son mari mort et enterré. Rien n'est cependant écrit sur son aspect physique (Dufournet, 37-42).

Dans De Gombert et des deux clers, Bodel procède différemment. Le paysan, messire Gombert, parfait imbécile [...], est croyant bien faire. Sa femme, dame Gille, est belle, gracieuse avec les yeux qui ont l'éclat du cristal. Quant au caractère, dame Gille est une brave femme. Ils ont une fille qui est décrite comme 'pucelle'. Nous connaissons donc le métier de l'homme avec deux petites remarques sur son caractère alors que la dame est décrite très soigneusement. Sa beauté entre même dans la morale du fabliau : "Aucun homme qui a une belle femme ne doit [...]

1 Le recueil de Jean Dufournet contient les fabliaux plutôt représentatifs qui figurent dans cet article. 
laisser un clerc coucher dans son hôtel. » (Dufournet, 48-55) Dans Brunain et la vache au prestre le couple n'est pas caractérisé, nous n'avons qu'un paysan et sa femme. La seule information est donnée par le prêtre qui appelle le paysan «sage » (Dufournet, 56-61). Haimet, Barat et Travers sont simplement identifiés comme malfaiteurs. Travers dans De Haimet et de Barat décide de revenir chez sa femme et recommencer à vivre honnêtement. Sa femme est désignée comme la dame «Marie qui avait su se débrouiller » et Travers s'exclame qu'il n'est ni fou ni joueur et qu'il se sent fort et dégourdi (Dufournet, 56-61)

Les quatre fabliaux de Jean Bodel que nous avons sélectionnés offrent une variété d'expression des marqueurs d'identité, aussi bien dans la manière que dans le degré. Bodel décrit les caractéristiques de ses personnages systématiquement, aléatoirement. Dans Le Villain de Bailleul il met un fort accent sur la laideur de l'homme, alors qu'il fait l'éloge de la beauté de la dame dans De Gombert et les deux clers. Dans ces deux cas, il insiste plus sur les caractéristiques physiques que sur le tempérament. Dans Brunain la vache au prestre et De Haiment et de Barat, il laisse la caractérisation presque complètement à part. Dans ces quatre fabliaux, le métier caractérise l'homme (paysan, clerc, malfaiteur) tandis que les femmes restent femmes ou dames.

\section{RUTEBEUF}

Penchons-nous maintenant sur deux fabliaux de Rutebeuf, De la dame qui fist trois tours entour le moustier et C'est li testament de l'asne. Dans le premier, l'homme (le bon écuyer) est décrit comme sot, débonnaire et naif (la femme " finit par lui faire croire que demain le ciel sera de cendre »). La femme n'est décrite que dans les jurons de l'écuyer : "espèce de grande salope, vraie putain. » Rutebeuf mentionne aussi une personne subsidiaire, appelée selon son métier - chambrière. Dans le premier fabliau il y a un clerc, dans le deuxième il y en a deux. Le prêtre est avare (" qui avait mis toute son application à accumuler revenus et biens »), mais l'évêque est défini bien différemment : « loin d'être cupide et avare, il était courtois et bien élevé ». Rutebeuf caractérise son personnage masculin dans De la dame qui fist trois tours entour le moustier par son métier et ses caractéristiques tandis que le personnage féminin n'est pas vraiment décrit. Il caractérise les deux clercs par les particularités de leur tempérament. (Dufournet, 330-348)

\section{HUON PIAUCELE, BOIVIN ET EUSTACHE D‘AMIENS}

Dans le fameux fabliau Estormi, Huon Piaucele raconte l'histoire des trois prêtres qui sont, selon la description, tous les trois privés de charme. L'écrivain les dépeint à l'aide des adjectifs " gras et gros, gras et énorme, affreusement 
laid ». Il insiste alors plus sur leur poids que sur leur perversion, qui est le thème du fabliau. Les prêtres essaient de séduire «la bonne dame » Yfame qui est également " une excellente épouse et très sage " (ses caractéristiques corporelles ne sont cependant pas mentionnées). Son mari, avec lequel elle vit dans la misère, s'appelle Jean, il est " un bel homme d'une grande robustesse ». Le héros du titre, Estormi, est le neveu de Jean, un « jeune homme» qui «se dirigea vers le prêtre $[\ldots]$ en homme qui n'était pas plus poltron que s'il était tout entier de fer ». Tout ce que Piaucele communique au lecteur (à vrai dire, à l'auditeur) sur les qualités d'Estormi tient dans cette citation, mais il ajoute dans la morale qui conclut le fabliau quelques informations, intéressantes pour notre recherche : " [...] croyez-vous que, quelle que soit sa pauvreté, une honnête femme se dévergonde ? - [et,] Quoi qu'il en soit, on ne doit pas, on ne doit pas à mon avis, mépriser un parent modeste, si pauvre soit-il, à moins que ce ne soit un traitre ou un brigand. Même s'il est fou ou joueur, il se range en fin de compte. [Il se range, et l'ordre du monde est préservé]» Une femme est honnête et un homme honorable quoi que soit son métier. (Dufournet, 257-287)

En comparaison avec Huon Piaucelle, Boivin est plus modeste en décrivant ses personnages. Dans De Boivin de Provins il parle d'un joyeux drille, Boivin, qui est un homme «fort rusé, maître en fourberie » mais un paysan selon le métier. L'autre personnage est Mabile qui se connaît en tromperie, plus « qu'aucune femme de l'endroit. » On s'aperçoit vite qu'elle est entremetteuse, même si elle n'est jamais nommée comme telle. (Dufournet, 236-253)

Eustache d'Amiens, en revanche, est plus généreux avec les marqueurs d'identité. Le héros de $D u$ boucher d'Abeville exerce le métier évoqué dans le titre du fabliau. Il est un boucher "sage, courtois, valeureux, honnête ; il rendait souvent de grands services à ses voisins pauvres et nécessiteux; il n'était ni avare ni cupide et loin d'être méchant et médisant ». La femme qu'il rencontre sur le chemin (personnage secondaire) est «brave et pauvre ». Elle lui suggère de trouver refuge chez le prêtre qui est un vrai contraste du boucher. Le doyen est «bouffi d'orgueil et un fort méchant personnage ». Le boucher l'accuse d'être un "chapelain insensé, coquin, un rustre et insatiable du bien d'autrui ». Il vit avec deux femmes, une servante (accusée par la dame d'être "voleuse et menteuse, garce et idiote etc. »). La dame, concubine du prêtre, est décrite comme " une dame fort jolie et très mignonne, yeux vifs et rieurs ». Elle est introduite une fois comme prêtresse. Dans le fabliau apparait aussi un " pastoureau ». Eustache d'Amiens décrit en détail le caractère des deux personnages masculins pendant qu'il se concentre sur l'aspect physique de la dame. L'écrivain fait apparaître les métiers des personnages (" doyen, boucher, pastoureau, servante ») et donne le nom " prêtresse » à l'amante de prêtre ce qui est intéressant, car nous aurions attendu une telle identification pour une épouse de quelqu'un qui exerce un certain métier. (Dufournet, 100-129) 
Entre Boivin, Huon Piaucelle et Eustache D'Amiens c'est ce dernier qui insiste le plus sur les marqueurs d'identité et le fait selon notre hypothèse : la femme est décrite comme «belle pretrêsse » et l'homme comme « sage boucher ».

\section{COURTEBARBE, HUON DE CAMBRAI ET GAUTIER LE LEU}

Comme Boivin, Courtebarbe, dans Les trois avugles de Compiegne, est très mesuré en décrivant les personnages. Les aveugles sont, tout simplement, aveugles, sans différenciation individuelle. Le clerc est « beau et élégant » (aspect physique), et il a un serviteur. Dans l'histoire il y a un personnage qui n'est marqué que par son métier - « l'aubergiste ou un tenancier ». (Dufournet, 314-329)

Un aubergiste apparaît aussi dans Del prestre taint de Gautier Le Leu, mais seulement dans l'introduction. Il raconte l'histoire du bourgeois (c'est la seule information que Le Leu nous donne sur lui) qui avait un prêtre pour voisin. Il avait aussi une femme "forte, courtoise, fraiche, gracieuse, belle et avisée ", nous voyons bien que son aspect physique épouse son caractère. En plus, l'histoire montre une entremetteuse et Hersent, marguillère de l'église. (Dufournet, 171-191)

La male Honte de Huon de Cambrai raconte l'histoire d'un « Anglais » qui était un « homme extraordinairement riche, habitait une ville ", mais il est décédé. Après sa mort, un «vilain » qui est « le héros de ce conte » doit amener une partie de son héritage au roi d'Angleterre. Le paysan nommé Honte est mal acquis à la cour. Il est décrit comme "l'homme brave même » dont le "discours et langage sont pleins d'effronterie et de sottises qu'au point de ne pas montrer trace de raison » et comme « fou débite des sottises » par un des chevaliers. Malgré tout, il persiste « sans méditer de ruse ni tromperie ». (Dufournet, 152-161)

Nous pouvons conclure que Gautier Le Leu décrit de manière plus détaillée la femme et laisse les descriptions des autres à part; Courtebarbe et Huon de Cambrai n'introduisent pas de personnages féminins et caractérisent peu ceux masculins.

\section{LES FABLIAUX ANONYMES}

Analysons encore six fabliaux anonymes: Du segretain moine, Des tresces, Baillet, Le dit des perdriz, Du prestre et du leu et Du prestre crucefié. Ils ont tous en commun un prêtre corrompu. Dans Du segretain moine on lit l'histoire d'une bourgeoise, Idoine, qui est « très vaillante et courtoise » et en même temps « élévée, modeste, courtoise et fine ». Le moine la décrit « comme la plus belle, la plus courtoise qui soit sur tout le territoire ». Son mari, qui se trouve brutalement attaqué dans les bois, s'appelle Guillaume, il est banquier, « habile dans son métier ». La dame est 
dépeinte selon plusieurs caractéristiques tandis que l'homme n'est "que" banquier. D'autres personnages sont mentionnés, marqués par leur métier : brigand, serviteur, veilleurs, servante (bonniche, la petite bonne), aubergiste, Thibaud le métayer. (Dufournet, 193-232)

Le fabliau Des tresces recourt à de nombreux superlatifs, car le fabliau raconte l'histoire d'un chevalier. Il est un individu « courageux, courtois, éloquent, sage, bref, plein de qualités, et qui recherche tellement la prouesse que jamais il ne voulut reculer devant aucune entreprise qui fut à sa portée ». Sa femme est une femme de grande naissance et une bonne chrétienne, cette femme est une bourgeoise qui en beauté est son parfait sosie. La description est donc faite des talents pour l'homme et des origines, de la foi et de la beauté pour la femme. (Dufournet, 288-231)

Les trois fabliaux qui nous restent contiennent tellement peu de marqueurs d'identité que nous pouvons les traiter en un seul paragraphe. Dans Baillet nous rencontrons un brave savetier qui a une " très belle femme " séduite par "prêtre lardier ». Le fabliau Du prestre crucefié est encore plus limité en ce qui nous intéresse. Il parle de monseigneur Roger, «le brave homme qui était passé maître dans l'art de sculpter des statues et de tailler des crucifix » et sa femme qui aimait un prêtre. Le dit de perdrix confronte un paysan, un prêtre et une dame " qui souhaitait non pas être très riche mais plutôt satisfaire tous ses désirs ». Notre dernier fabliau, et le plus court entre ceux que nous avons traités, est Du prestre et du leu. Lui aussi parle d'une dame, d'un prêtre, d'un vilain et d'une personne caractérisée par son métier - une servante. (Dufournet, 91-99, 162-167, 140-149).

L'analyse des fabliaux montre qu'il n'existe aucune règle générale pour le genre des fabliaux sur comment et à quel point les personnages doivent être caractérisés. Certains se contentent de les décrire avec un mot : « le paysan » et " une dame» (Le dit de perdrix), tandis que d'autres ajoutent des descriptions plus ou moins longues. Nous voyons aussi que, même dans les fabliaux où les caractères sont moins élaborés, l'homme est toujours nommé par son métier tandis que la femme souvent reste 'femme' ou 'dame' (par exemple Du prestre et $d u$ leu). Les auteurs insistent en général plus sur l'aspect physique et émotionnel des femmes que des hommes.

\section{ANALYSE QUANTITATIVE}

Jetons un regard d'analyse quantitative sur notre hypothèse. Nous allons compter les marqueurs d'identité dans dix-huit fabliaux. Notre analyse comprendra plusieurs catégories : les personnages qui sont caractérisés selon leur aspect physique/leur tempérament/leur métier et ces mêmes catégories pour les personnages 
féminins. On commence par les personnages principaux sans clercs, pour ensuite ajouter les clercs et, enfin, compter tous ensemble. Nous mentionnerons également les personnages secondaires qui sont parfois identifiés par un métier mais nous les traiterons séparément. En fonction des chiffres obtenus, nous essaierons de formuler quelques conclusions.

Tableau $1:$ Analyse des personnages principaux

\begin{tabular}{|l|l|l|l|}
\hline $\begin{array}{l}\text { Nombre des } \\
\text { personnages } \\
\text { (tous) }\end{array}$ & $\begin{array}{l}\text { Personnages masculins } \\
\text { avec les marqueurs } \\
\text { d'identité pour l'aspect } \\
\text { physique }\end{array}$ & $\begin{array}{l}\text { Personnages masculins } \\
\text { avec les marqueurs } \\
\text { d'identité pour le } \\
\text { tempérament }\end{array}$ & $\begin{array}{l}\text { Personnages masculins } \\
\text { avec un métier déterminé }\end{array}$ \\
\hline 46 & 7 & 13 & 29 \\
\hline $\begin{array}{l}15 \text { femmes, } \\
31 \text { hommes }\end{array}$ & $\begin{array}{l}\text { Personnages féminins } \\
\text { avec les marqueurs } \\
\text { d'identité pour l'aspect } \\
\text { physique }\end{array}$ & $\begin{array}{l}\text { Personnages féminins } \\
\text { avec les marqueurs } \\
\text { d'identité pour le } \\
\text { tempérament }\end{array}$ & $\begin{array}{l}\text { Personnages féminins } \\
\text { avec un métier déterminé }\end{array}$ \\
\hline & 6 & 9 & $2^{*}$ \\
\hline
\end{tabular}

Commentaire : à partir des données, nous observons que les auteurs des fabliaux choisis insistent plus sur les descriptions de tempérament pour les deux sexes sans différences. Le résultat est presque égal. Quant aux marqueurs de l'aspect physique, il y a une légère préférence du côté des femmes. Nous constatons que les écrivains décrivent presque à la même fréquence les caractéristiques corporelles et le tempérament pour les deux sexes. Une grande différence apparaît pour la caractérisation selon les métiers. Quinze personnages masculins ont un métier alors qu'il n'y a que deux femmes dont une pas même caractérisée directement (l'entremetteuse Mabile de De Boivin de Provins).

Tableau 2: Analyse pour les clercs

\begin{tabular}{|l|l|l|}
\hline Nombre des clercs(tous) & $\begin{array}{l}\text { Clercs avec les marqueurs } \\
\text { d'identité pour l'aspect } \\
\text { physique }\end{array}$ & $\begin{array}{l}\text { Clercs avec les marqueurs } \\
\text { d'identité pour le } \\
\text { tempérament }\end{array}$ \\
\hline 14 & 3 & 6 \\
\hline
\end{tabular}

Commentaire : Nous voyons que la caractérisation des clercs est de moindre importance que celle des laïcs. Il semble que cela va de soi qu'un prêtre de fabliau soit corrompu, glouton ou dévergondé et qu'ainsi, les marqueurs d'identité ne soient pas nécessaires. Toutefois, certains fabliaux soulignent l'aspect physique des clercs et, plus souvent, leur tempérament. 
Tableau 3 : Analyse pour les personnages principaux et les clercs

\begin{tabular}{|l|l|l|l|}
\hline $\begin{array}{l}\text { Nombre des } \\
\text { personnages } \\
\text { (tous) }\end{array}$ & $\begin{array}{l}\text { Personnages masculins } \\
\text { avec les marqueurs } \\
\text { d'identité pour l'aspect } \\
\text { physique }\end{array}$ & $\begin{array}{l}\text { Personnages masculins } \\
\text { avec les marqueurs } \\
\text { d'identité pour le } \\
\text { tempérament }\end{array}$ & $\begin{array}{l}\text { Personnages masculins } \\
\text { avec un métier } \\
\text { déterminé2 }\end{array}$ \\
\hline 32 & 4 & 10 & 15 \\
\hline $\begin{array}{l}15 \text { femmes, } \\
17 \text { hommes }\end{array}$ & $\begin{array}{l}\text { Personnages féminins } \\
\text { avec les marqueurs } \\
\text { d'identité pour l'aspect } \\
\text { physique }\end{array}$ & $\begin{array}{l}\text { Personnages féminins } \\
\text { avec les marqueurs } \\
\text { d'identité pour le } \\
\text { tempérament }\end{array}$ & $\begin{array}{l}\text { Personnages féminins } \\
\text { avec un métier déterminé }\end{array}$ \\
\hline & 6 & 9 & $2^{*}$ \\
\hline
\end{tabular}

Commentaire : Si nous ajoutons les clercs à nos premiers résultats, la comparaison des chiffres des identités entre les sexes deviennent moins pertinentes étant donné que cela résulte en un chiffre plus élevé de personnages masculins que féminins. La différence entre les hommes caractérisés par un métier et les femmes qui ne le sont pas est encore plus évidente.

Malgré le fait que les auteurs des fabliaux négligent presque complètement les professions des leurs personnages féminins principaux, ils les nomment plus souvent quand elles jouent les rôles des personnages secondaires. Dans nos fabliaux choisis, nous trouvons plusieurs servantes, une chambrière et une bonne (bonniche). Dans le Bouchier d'Abeville nous rencontrons une prêtresse. Une telle identification apparaît dans les fabliaux (hors de ceux que nous avons choisis), les femmes portent la forme féminine du métier que leur mari exerce. Le cas de prêtresse semble intéressant, car la femme est la concubine du clerc. Les personnages secondaires masculins sont également marqués par leurs métiers, nous pouvons citer les métiers suivants : pastoureau, serviteur, métayer, veilleur, brigand et aubergiste.

\section{CONCLUSION}

Dans la présente recherche nous avons essayé de vérifier l'hypothèse selon laquelle les hommes dans les fabliaux sont identifiés par ce qu'ils font, par le métier qu'ils exercent et moins souvent par leurs caractéristiques physiques, et que les femmes sont soit identifiées par leur mari, soit par leurs propres caractéristiques. Les conclusions sont les suivantes :

2 J'ai pris en compte les trois aveugles (Des trois aveugles de Compiegne) et trois malfaiteurs (De Haimet et de Barat) étant donné qu'ils fonctionnent comme un collectif. Comme métiers, en outre, j’ai pris en considération les paysans (vilains) et un cas de chevalier (pour le différencier de l'identification générale - homme). 
1) Il n'existe pas de système, de règle selon laquelle les auteurs des fabliaux caractérisent leurs personnages. La quantité des informations que le lecteur obtient sur l'identité d'un personnage des fabliaux varie, l'écrivain décide à chaque fois comment et avec combien de marqueurs d'identité il va définir son personnage. Cette absence de règle générale s'accorde avec la richesse rendue possible par la diversité des fabliaux, ce qui représentait un problème aux historiens de la littérature quand ils définissaient le genre.

2) L'analyse quantitative de dix-huit fabliaux a prouvé que les auteurs ne différencient pas les genres selon la quantité de marqueurs d'identité. L'analyse a montré que les auteurs décrivent presque aussi souvent les caractéristiques des personnages masculins et féminins. Les prêtres sont moins souvent sujets à une caractérisation explicite que les laïcs.

3) L'analyse a montré aussi que les personnages masculins principaux sont souvent caractérisés selon leur métier alors que les personnages féminins, dans la grande majorité des cas, ne portent pas de marqueur du métier. C'est différent pour les personnages secondaires où les hommes et les femmes sont aussi souvent caractérisés avec un métier les uns que les autres.

La recherche a partiellement confirmé notre hypothèse. Il est vrai que les hommes dans les fabliaux sont identifiés par ce qu'ils font, mais ils sont également caractérisés par leur aspect physique et leur tempérament. Les femmes qui font partie des personnages principaux sont aussi caractérisées par le physique et le tempérament, très rarement par un métier. La caractérisation des personnages secondaires est plus " égalitaire », car les hommes et les femmes sont nommés par un métier. Il est difficile d'affirmer que les constatations tirées de l'analyse de dix-huit fabliaux soient valables pour l'ensemble des fabliaux, bien que nous ayons sélectionné des fabliaux particulièrement diversifiés pour notre recherche. On sera toujours surpris de trouver, au plus profond du Moyen âge, des événements et des personnages qui tranchent totalement sur ce que l'on a appris de cette époque-là. Il est si facile d'oublier que l'on a appris de la littérature et étudié des livres et de vieux documents poussiéreux d'où on tire, chaque fois de nouveau, un Moyen âge recréé où l'on se reconnaît dans ce que l'on en sait, pas moins que dans ses lacunes et, finalement, dans la confabulation qui les remet en rapport (réciproque) et leur confère un sens. Le mot (con)fabulatio comprend fabula, l'afr. fable... qui n'est pas loin du fablel, «fabliau ». Avec le temps, nous devenons notre imagination, force imaginatrice dématérialisée qui se traduit dans l'espace par la parole. Le corps, signe imaginé, lacéré de saint, ou cet autre, perverti et débauché. Confabulation, parole imaginaire, lacune et manque de vie, là précisément où elle devrait se montrer comme la vie elle-même. Dans toute sa fragilité, et du moins pour sauvegarder les apparences, la valeur de celle-ci ne dépend-elle pas, alors, de la confabulation elle-même ? 


\section{LITTÉRATURE}

Crocker, Holly A., Comic Provocations : Exposing the corpus of old French Fabliaux, New York, Palgrave Mac Millan (2006).

Pearcy, Roy J., Logic and Humour in the fabliaux, Cambridge, D. S. Brewer (2007). Stearns Schenck, Mary Jane, The didacticism of the Fabliau dans THE FABLIAUX Tales of Wit and Deception. Amsterdam/Philadelphia, John Benjamins Publishing Company (1987) 19-36.

Horton, Ingrid, Engendering Vice: The Exemplarity of the Old French Fabliaux, Lawrence, University of Kansas (2007).

Wehner Eaton, Ellen, Shame culture or guilt culture : the evidence of the medieval French fabliaux, Toronto: University of Toronto (2000).

Muñoz, Natalie, Disabusing Women in the Old French Fabliaux, New York, Peter Lang Publishing (2014).

Bégin, Clarissa, Le paroxysme de la violence dans l'érotisme des fabliaux, Toronto,. The University of Western Ontario (1999).

Collart, Marie-France, L'univers de la prostitution dans les fabliaux et sa représentation, Valencia: Universitat de València (2012).

de Looze, Laurence, Sex, Lies, and Fabliaux: Gender, Scribal Practice, and Old/ new Philology in "Du Cheualier Qui Fist Les Cons Parler", The Romantic review (85/4; 1994).

Jacobs, Thibault, Sociale realities in de Franse fabliaux. Een statistische benadering, Leidschrift $(25 / 3 ; 2010)$.

Dufournet, Jean, Fabliaux du Moyen Age, Paris, Flammarion (1998)

Urb Ferlež

Université de Ljubljana ferlez.urh@gmail.com

Miha Pintarič

Université de Ljubljana Miha.Pintaric@ff.uni-lj.si

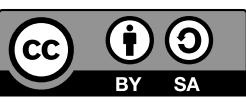




\section{Identifikacijska znamenja moških in ženskih likov v fabliaujih}

Članek obravnava identifikacijska znamenja moških in žensk v fabliaujih. Preverja hipotezo, po kateri naj bi bili moški liki v fabliaujih indentificirani po tistem, kar počnejo, tj. po poklicu ki ga opravljajo, redkeje pa po svojih telesnih in karakternih značilnostih. Po drugi strani naj bi bili ženski liki identificirani bodisi po zakonskih možeh bodisi po telesnih značilnostih in značaju. Kvalitativna in kvantitativna analiza osemnajstih izbranih fabliaujev sta pokazali, da se količina informacij, ki jo lahko bralec dobi o identiteti nekega lika, spreminja od fabliauja do fabliauja, tudi znotraj opusov enega pisca (npr. Rutebeufa). Pokazalo se je tudi, da pisci ne razlikujejo spolov glede na to, kako in koliko opisujejo njihovo zunanjost in značaj. Po drugi strani so moški liki pogosto označeni s poklicem, ženski pa v veliki večini primerov to niso. Ženske stranske osebe so pogosteje označene s poklicem kot glavne.

Ključne besede: fabliauji, identifikacijska znamenja, ženske in moški v srednjem veku, Rutebeuf, Jean Bodel. 